production of pro-inflammatory cytokines were analyzed in urinary myeloid cells of patients with LN.

Results: Myeloid cells were identified by the expression of CD45+ and CD11C+ in the urine of patients with LN. The frequency and absolute numbers of myeloid cells were markedly increased in the urine of patients with proliferative $L N$ than non-proliferative LN. In addition, titers of anti-dsDNA antibodies were correlated with the frequency or numbers of urinary CD11c+ myeloid cells. These urinary CD11c+ myeloid cells showed the phenotypes of infiltrated monocyte-derived cells rather than tissue-resident macrophage. In addition, CD11c+ myeloid cells were localized in tubulointerstitium and had capacity to produce pro-inflammatory cytokines including IL-6. Further, we found that there was a significant population of tubule cells in the urine, which is correlated with the frequency of CD11c+ myeloid cells.

Conclusion: Our results indicate that CD11c+ myeloid cells are present in the urine and contribute to tubulointerstitial inflammation in proliferative LN

Disclosure of Interests: None declared

DOI: 10.1136/annrheumdis-2019-eular.2528

\section{AB0175 1 ABSOLUTE REDUCTION OF PERIPHERAL CD4+CD25 +FOXP3+T REGULATORY CELLS IN PATIENTS WITH SYSTEMIC LUPUS ERYTHEMATOSUS}

Xiaoqing Liu, Yanan Duan, Nalin Lai, Gaiqin Chai. The Second Hospital of Shanxi Medical University, Department of Rheumatology, Taiyuan, China

Background: $\mathrm{T}$ lymphocytes are important contributors to systematic lupus erythematosus (SLE). Regulatory T (Treg) cells, with the capacity to suppress immune responses, effector $\mathrm{T}$ cells (Teff), which promote inflammation, have been intensively studied in recent years. However, previous reports describing the respective changes of Treg and Teff, especially T helper cells (Th17) in SLE patients were controversial. Here, we investigated both absolute number and percentage of CD4+CD25+Foxp3+Treg (CD4Treg) cells and effector cells on a large scale and the role of low-dose interleukin-2 (IL-2) in SLE.

Objectives: To investigate both absolute number and percentage of CD4+CD25 +Foxp3+Treg (CD4Treg) cells and effector cells on a large scale and the role of low-dose interleukin-2 (IL-2) in SLE.

Methods: Two hundred and thirty-five SLE patients (219 women and 16 men), with mean age of $37.80 \pm 14.00$ years, were enrolled. The absolute count and percentage of subpopulation of peripheral blood (PB) lymphocyte in these patients were measured by flow cytometry combined with internal microsphere standard. And low-dose IL-2 was used among 127 patients at a dosage of fifty WIU every day for five days. Immunological and clinical assessments were performed again at the end of IL-2 treatment. Ninety healthy volunteers, matched for patients' age and gender, were also included for the estimation of lymphocyte subsets.

Results: As compared to healthy controls (median of Treg cells: 33.09 cells/ul), the absolute number of circulating CD4Treg cells were significantly decreased in SLE patients (median: 15.49 cells/ul, $\mathrm{P}<0.001$ ). The median ratios of Th17/Treg cells in patients were greatly higher than those of healthy volunteers $[0.42(0.19$, $0.88)$ vs. $0.21(0.15,0.34), P<0.001]$, while there was not significantly different in peripheral Th17 cell between two groups. Besides, Th1, Th2, CD8+T, B cells and their respective ratios to Treg cells were like that of Th17 cells as well. Moreover, CD4Treg cells were negatively correlated with ESR and SLEDAI score $(r=-0.198$, $P=0.01 ; r=-0.25, P=0.002)$. While no obvious correlation was seen between Th17 cells and SLEDAI score. After IL-2 therapy in SLE, there was a four-fold increase in circulating CD4Treg cells $[43.73(24.08,74.22)$ vs. $11.95(7.51,20.34)$, $\mathrm{P}<0.001$ ], whereas Th17 cells were increased slightly. The ratio of Th17/Tregs was decreased significantly in patients with IL-2 treatment $[0.19(0.09,0.41)$ vs. $0.52(0.23,0.95), P<0.001]$, tended to balance and had no difference with healthy individual $(P=0.275)$. Similarly, there were same trends in Th1, Th2, CD8+T, B and NK cells.

Conclusion: The reduction of CD4Tregs but not the elevation of effector cells may be the major reason for imbalance of Teff/Treg, indicating that SLE is an autoimmune disease triggered by the defect of immunotolerance. More importantly, low-dose IL-2 might promote the proliferation of various lymphocyte subpopulation, and mainly modulated the abundance and immunosuppression activity of Tregs, which effectively induced autoimmune tolerance and further improved clinical symptoms.

\section{REFERENCES}

[1] Bonelli M, Goschl L, Bluml S, et al. (2014) CD4+CD25-Foxp3+T cells: a marker for lupus nephritis? Arthritis Research \& Therapy. 16: R104.

[2] M Miyara, G Gorochov, M Ehrenstein, et al. (2011) Human FoxP3+ regulatory $\mathrm{T}$ cells in systemic autoimmune diseases. Autoimmunity Reviews. 10 (12): 744-55

[3] Żabińska M, Krajewska M, Kościelska-Kasprzak K, et al. (2016) CD4(+) CD25(+) CD127(-) and CD4(+)CD25(+)Foxp3(+) Regulatory T Cell
Subsets in Mediating Autoimmune Reactivity in Systemic Lupus Erythematosus Patients. Arch Immunol Ther Exp (Warsz). 64(5): 399-407.

Disclosure of Interests: None declared

DOI: 10.1136/annrheumdis-2019-eular.2726

\section{AB0176 MITOGEN- AND STRESS-ACTIVATED PROTEIN KINASE- 1 (MSK1) AS THE LINK BETWEEN MIR-130A- DYSREGULATION AND CDC2-ACTIVATION IN SJÖGREN'S SYNDROME}

Ana P. Lopes ${ }^{1}$, Joel van Roon ${ }^{1}$, Sofie Blokland ${ }^{1}$, Maojie Wang ${ }^{2}$, Eleni Chouri ${ }^{1}$, Aike A. Kruize ${ }^{3}$, Boudewijn Burgering ${ }^{2}$, Marzia Rossato ${ }^{1}$, Timothy R. Radstake ${ }^{1}$, Maarten Hillen'. ' University Medical Center Utrecht, Department of Rheumatology and Clinical Immunology, Laboratory of Translational Immunology, Utrecht, Netherlands; ${ }^{2}$ University Medical Center Utrecht, Department of Molecular Cancer Research, Center Molecular Medicine, Oncode Institute, Utrecht, Netherlands; ${ }^{3}$ University Medical Center Utrecht, Department of Rheumatology and Clinical Immunology, Utrecht, Netherlands

Background: Primary Sjögren's syndrome (pSS) is a systemic autoimmune disease characterized by lymphocytic infiltration of the exocrine glands and dryness of mouth and eyes. T and B lymphocytes that infiltrate the salivary glands play a central role in local production of autoantibodies and cytokines, associated with dryness and tissue-damage. Type- 2 conventional dendritic cells (cDC2) are very potent antigen-presenting cells and have the ability to produce a variety of cytokines involved in T and B cell activation, germinal centres formation and autoantibody production.

Objectives: Considering the critical role of microRNAs (miRNAs) in regulation of cell activation, we investigated their potential dysregulation in circulating $c D C 2 s$ of patients with pSS compared to healthy controls $(\mathrm{HC})$.

Methods: CD1c-expressing cDC2s were isolated from peripheral blood of pSS patients and controls from two independent cohorts. In the donors from the discovery cohort (15 pSS, $6 \mathrm{HC}$ ) the expression of 758 miRNAs was screened; the replication cohort (14 pSS, $11 \mathrm{HC}$ ) was used to confirm consistent differential expression of 18 identified miRNAs. A quantitative mass spectrometry-based technique (pSILAC) in HEK-293T cells was used to identify novel targets of the replicated miRNAs. Target-miRNA interaction was replicated in primary cDC2s. Differences in cytokine production between pSS and $\mathrm{HC}$ cDC2s were evaluated by FACS. cDC2s were cultured in the presence of different MSK1-inhibitors to examine their effect on cytokine production.

Results: The expression of miR-130a and miR-708 was consistently decreased in cDC2s from pSS patients compared to $\mathrm{HC}$ in both cohorts, and both miRNAs were downregulated upon stimulation via TLR3 and TLR7/8. MSK1 was identified as a novel target of miR-130a and overexpression of miR-130a reduced MSK1 protein expression in both HEK-293T cells and primary cDC2s. In-line with the regulation of MSK1 by miR-130a, MSK1 expression was higher in cDC2s of pSS patients as compared to controls. An increased frequency of $\mathrm{CDC} 2 \mathrm{~s}$ producing-IL12 and TNF- $\alpha$ was observed in pSS patients compared to $\mathrm{HC}$, consistent with the central role of MSK1 in regulation of cytokine production. Exposure to either of two different MSK1 inhibitors in vitro reduced $\mathrm{CDC} 2$ activation and the production of IL-12, TNF- $\alpha$ and IL-6.

Conclusion: We here provide the first evidence of molecular dysregulation of cDC2s in pSS, including decreased expression of miR-708 and miR-130a, which can result from TLR activation, and enhanced production of pro-inflammatory cytokines. In view of its central role in NF-KB signalling, inhibition of MSK1 to decrease cell activation and inhibit pro-inflammatory cytokine production represents a novel therapeutic avenue for treatment of Sjögren's Syndrome.

Disclosure of Interests: None declared

DOI: 10.1136/annrheumdis-2019-eular.6806

\section{AB0177 ARE THERE ANY CLINICAL AND SEROLOGICAL DIFFERENCES BETWEEN PATIENTS WITH PRIMARY AND SECONDARY (SLE) ANTIPHOSPHOLIPID SYNDROME?}

Magdalena Dryglewska ${ }^{1}$, Andrzej Majdan ${ }^{2}$, Maria Majdan ${ }^{1} .{ }^{1}$ Medical University of Lublin, Dept of Rheumatology and Connective Tissue Diseases, Lublin, Poland; ${ }^{2}$ Medical University of Lublin, Dept of Gynaecological Oncology and Gynecology, Lublin, Poland

Background: Antiphospholipid syndrome (APS) could be diagnosed as primary (PAPS) or secondary (SAPS - accompanying other diseases, mainly found in patients with systemic lupus erythematosus -SLE)

Objectives: The aim of our study was to determine if pts with PAPS and SAPS had different clinical and serological status. 
Methods: The study involved 80 (59-f and 21-m) APS patients (pts) 24 pts had PAPS and 56 pts had APS following SLE. In the whole study group the mean age was: $39.3 \pm 12.7$ years (range 18-71), the duration of the disease was $8.8 \pm 8.3$ years (range 0-37).

The presence of aPL was detected in patients' serum using the commercially available tests: aPL-immunodot assay Anti-Phospholipid 10 Dot, for the qualitative detection of $\lg G$ or $\lg M$ antibodies. The statistical data analysis was performed using Statistica v13.0

Results: In the study group of $\mathbf{8 0}$ patients we have detected the presence of the following aPLs: a- cardiolipin IgM $-37 \%$, IgG $-50.6 \%$; a-phosphatidic acid IgM -19.7\%, IgG -13.3\%; a-phosphatidylcholine IgM and IgG -1.2; a-phosphatidylethanolamine $\lg \mathrm{M}-1.2 \%$ and $\lg \mathrm{G}-0$; a-phosphatidylglycerol $\lg \mathrm{M}-5.0 \%$, $\lg \mathrm{G}$ 12.5\%; a-phosphatidylinositol IgM-10.0\%, IgG-12.5\%; a-phosphatidylserine IgM$31.0 \%$, IgG-50.6\%; a-annexin V IgM -20\%, IgG -10\%; a-ß2-GP I lgM-44.5\%, IgG$35.0 \%$; a-prothrombin IgM -44.5\%, lgG-30\%.

Table 1

\begin{tabular}{|c|c|c|c|}
\hline & $\begin{array}{c}\text { PAPS } \\
n=24\end{array}$ & $\begin{array}{c}\text { SAPS } \\
\mathrm{n}=56\end{array}$ & $\mathrm{P}<0.05$ \\
\hline \multirow[t]{2}{*}{$\mathrm{aCL} \lg \mathrm{M}$ aCL $\lg \mathrm{G}$} & $\begin{array}{c}10 \\
(41.6 \%)\end{array}$ & $\begin{array}{c}20 \\
(35.7 \%)\end{array}$ & NS \\
\hline & $\begin{array}{l}10 \\
(41.6 \%)\end{array}$ & $\begin{array}{c}31 \\
(55.3 \%)\end{array}$ & NS \\
\hline a-phosphatidic acid IgM a-phosphatidic acid IgG & $\begin{array}{c}8(33.3 \%) \\
3(12.5 \%)\end{array}$ & $\begin{array}{c}8(14.3 \%) \\
8 \\
(14.3 \%)\end{array}$ & $\begin{array}{c}P=0.0515 \\
N S\end{array}$ \\
\hline a-phosphatidylcholine IgM a-phosphatidylcholine $\lg G$ & $\begin{array}{c}0(0 \%) \\
0(0 \%)\end{array}$ & $\begin{array}{l}1(1.8 \%) \\
1(1.8 \%)\end{array}$ & $\begin{array}{l}\text { NS } \\
\text { NS }\end{array}$ \\
\hline \multirow{3}{*}{$\begin{array}{l}\text { a-phosphatidylethanolamine } \lg M \text { a- } \\
\text { phosphatidylethanolamine } \lg G \\
\text { a-phosphatidylglycerol } \lg \text { a-phosphatidylglycerol IgG }\end{array}$} & $0(2.2 \%)$ & $0(\%)$ & NS \\
\hline & $0(\%)$ & $0(\%)$ & NS \\
\hline & $\begin{array}{c}2(8.3 \%) \\
3(12.5 \%)\end{array}$ & $\begin{array}{c}2(3.6 \%) \\
7 \\
(12.5 \%)\end{array}$ & $\begin{array}{l}\text { NS } \\
\text { NS }\end{array}$ \\
\hline a-phosphatidylinositol IgM a-phosphatidylinositol lgG & $\begin{array}{l}5(20.8 \%) \\
3 \\
(12.5 \%))\end{array}$ & $\begin{array}{c}3(5.4 \%) \\
7 \\
(12.5 \%)\end{array}$ & $\begin{array}{c}P=0.0345 \\
N S\end{array}$ \\
\hline \multirow[t]{2}{*}{ a-phosphatidylserine $\lg M$ a-phosphatidylserine $\lg G$} & $\begin{array}{c}11 \\
(45.8 \%)\end{array}$ & $\begin{array}{c}14 \\
(25.0 \%)\end{array}$ & 0,0655 \\
\hline & $\begin{array}{l}12 \\
(50.0 \%)\end{array}$ & $\begin{array}{c}29 \\
(51.8 \%)\end{array}$ & NS \\
\hline \multirow[t]{2}{*}{ a-B2GPI IgM a-B2GPI IgG } & $\begin{array}{c}17 \\
(70.8 \%)\end{array}$ & $\begin{array}{c}12 \\
(28.6 \%)\end{array}$ & $P=0,0004$ \\
\hline & $7(29.2 \%)$ & $\begin{array}{c}21 \\
(37.5 \%)\end{array}$ & NS \\
\hline \multirow[t]{2}{*}{ a-annexin $\mathrm{V} \lg \mathrm{M}$ a-annexin $\mathrm{V} \lg \mathrm{G}$} & $6(25.0 \%)$ & $\begin{array}{c}10 \\
(17.9 \%)\end{array}$ & NS \\
\hline & $2(8.3 \%)$ & $6(10.7 \%)$ & NS \\
\hline \multirow[t]{2}{*}{ a-prothrombin IgM a-prothrombin IgG } & $\begin{array}{c}16 \\
(66.7 \%)\end{array}$ & $\begin{array}{c}20 \\
(35.7 \%)\end{array}$ & $P=0.0108$ \\
\hline & $8(33.3 \%)$ & $\begin{array}{c}16 \\
(28.6 \%)\end{array}$ & NS \\
\hline
\end{tabular}

Table 2

\begin{tabular}{lcc}
\hline Clinical symptoms & PAPS $\mathbf{n = 2 4}$ & SAPS $\mathbf{n = 5 6}$ \\
\hline stroke & $3(12.5 \%)$ & $5(9.0 \%)$ \\
pulmonary embolism & $\mathrm{NS}$ & \\
thrombosis & $6(25.0 \%)$ & $7(12.5 \%)$ \\
obstetric complications & $14(58.3 \%)$ & $19(34.0 \%)$ \\
valve defects & $\mathrm{NS}(18.9 \%)$ & $12 /(28.0 \%)$ \\
livedo reticularis & $\mathrm{NS}$ & $8(14.3 \%)$ \\
nephropathy & $4(16.7 \%)$ & $7(12.5 \%)$ \\
deep vein thrombosis & $\mathrm{NS}(8.3 \%)$ & $19(34.0 \%)$ \\
hypertension & $\mathrm{NS}$ & $19(34.0 \%)$ \\
convulsions/chorea & $5(20.8 \%)$ & $23(41.0 \%)$ \\
migrene & $\mathrm{NS}(58.3 \%)$ & $5(8.9 \%)$ \\
depression & $\mathrm{P}=\mathbf{0 , 0 4 2 2}$ & $4(8.9 \%)$ \\
\hline & $12(50.0 \%)$ & $2(3.6 \%)$ \\
\hline
\end{tabular}

The following clinical symptoms of APS were also present: stroke $-10 \%$, arterial/venous thrombosis $-34 \%$, pulmonary embolism- $16.3 \%$, obstetric complications-25.4\%, nephropathy - 30.0\%, hypertension- $43.8 \%$, valve defects $15.0 \%$, livedo reticularis $11.2 \%$, convulsions/chorea- $6.2 \%$, thrombocytopenia- $23.7 \%$ of the study group.

During the study we detected statistically significant differences in the frequency of occurrence of aPLs between groups with PAPS and SAPS: a-phosphatidylinositol $p=0,0345$; $a-\beta 2-G P$ | IgM $p=0.004$; $a$-prothrombin $\operatorname{lgM} p=0.0108$ a-phosphatidic acid lgM $\mathrm{p}=0.045$ (table 1)

There are no differences in the assessed clinical symptoms between pts with PAPS and SAPS, apart from significantly higher frequency of the deep vein thrombosis in pts with PAPS (p-0.04).

Conclusion: The clinical pictures of PAPS and SAPS are similar. Only deep vein thrombosis occurs more frequently in pts with PAPS.

Some classic and novel aPLs occur more frequently in pts with PAPS than in SAPS, and maybe could become a biomarker of PAPS

Disclosure of Interests: Magdalena Dryglewska: None declared, Andrzej Majdan: None declared, Maria Majdan Speakers bureau: MSD, UCB, Abbvie, Roche DOI: 10.1136/annrheumdis-2019-eular.1944

\section{$\mathrm{AB} 0178$ \\ TRANSCRIPTION PROFILING OF PERIPHERAL B CELLS IN ANTIBODY-POSITIVE PRIMARY SJOGREN'S SYNDROME REVEALS INTERFERON SIGNATURE AND UPREGULATED EXPRESSION OF GENES WHICH MAY BE PREDICTIVE OF TRANSFORMATION TO LYMPHOMA AND CORRELATED TO CHRONIC PULMONARY NON- TUBERCULOUS MYCOBACTERIAL (NTM) INFECTION}

Mehrnaz Maleki-Fischbach, Fatjon Leti, Brian O'connor, Tasha Fingerlin. National Jewish Health, Denver, United States of America

Background: Primary Sjögren's syndrome (pSS) is the second most common systemic autoimmune disease (after RA), with a prevalence of about $0.5 \%$ in the general population and it occurs primarily in women at a ratio of women to men of 9:1. Key features of the disease include infiltration of salivary and lacrimal glands by lymphocytes, production of inflammatory cytokines, and the production of autoantibodies. Nevertheless, systemic manifestations can arise in a proportion of pSS with pulmonary manifestation and higher incidence of NTM infection. Also, Bcell non-Hodgkin lymphoma $(\mathrm{NHL})$ development represents a severe complication, afflicting approximately $5 \%$ of patients. The risk of $\mathrm{NHL}$ occurrence in the setting of pSS, has been previously estimated to be 7-19 fold higher than the genera population. B activation is one of the proposed hypotheses, that accord with the known biological findings, including hypergammagolubinemia, presence of autoantibodies including SSA/Ro60/TROVE2, SSA/Ro52/TRIM21 and SSB/La48

Objectives: $B$ cells play a key role in the pathogenesis of pSS. The aim of this study was to analyze the transcriptome of B cells from patients with pSS and healthy controls to decipher the B cell-specific contribution to pSS.

Methods: RNA of negatively selected B cells from 5 ANA, SSA (both Ro60 and Ro 52), SSB and rheumatoid factor positive untreated female patients with pSS and 5 healthy controls was subjected to whole transcriptome sequencing. A false discovery rate corrected significance threshold of $a<0.1$ was applied to define differential gene expression.

Results: RNA-sequencing identified 56 and 23 up and down differentially expressed genes, respectively, and hierarchal clustering showed a clear separation between the two groups. Ingenuity Pathway Analysis revealed that these genes may play a role in interferon signaling (IFIT3, IFIT1, OAS1, MX1, STAT2 IFI35, IFITM1, ISG15, IFI27, IFI44L, IFI44, and PARP9), chronic mycobacteria infection (IFI35,IFI44,IFI44L,IFIT1,IFIT3,IFITM1,MX1,OAS1,OAS2,OAS3, STAT2) and transformation to myeloproliferative disorders, especially B cell lymphomas (CD160, CD38, ENAM, FUT4, GADD45B, IFI35, IFI44, IFI44L, IFIT3, IFITM1, ISG15, JCHAIN, MX1, OAS1, OAS2, OASL, RGS16, RNF213, RSAD2, SARS2, SATB1, SMAD7, and STAT2).

Conclusion: Upregulated expression of type I and type II interferon (IFN) induced genes was observed, establishing an IFN signature in pSS B cells. There are also genes that may play a role in other concomitant conditions like mycobac terial/NTM infection and higher risk for developing myeloproliferative conditions. This adds insight into the autoimmune process and suggests potential targets for future functional and prognostic studies.

\section{REFERENCES}

[1] Gottenberg, J.-E. Et al. Serum levels of $\beta 2$-microglobulin and free light chains of immunoglobulins are associated with systemic disease activity in primary Sjögren's syndrome. PLoS ONE 2013

[2] Schulte-Pelkum J., Fritzler M., and Mahler M.: Latest update on the Ro/ SS-A autoantibody system. Autoimmun Rev 2009 